\title{
The role of LMNB1 protein in replicative senescence of primary human fibroblasts
}

\author{
Morozova E. ${ }^{1 *}$, Laktionov P. ${ }^{1,2}$, Singh P. ${ }^{1,3}$ \\ 1 Novosibirsk State University, Novosibirsk, Russia \\ 2 Institute of Molecular and Cellular Biology, SB RAS, Novosibirsk, Russia \\ 3 Nazarbayev University School of Medicine, Nur-Sultan, Republic of Kazakhstan \\ *e-mail:morozovae613@gmail.com
}

Key words: senescence, lamin B1, CRISPR/Cas9

Motivation and Aim: Aging is a natural process that leads to a gradual decrease in the afunctional capabilities of the organism. Cellular senescence is tightly linked with general organism ageing, as senescent cells accumulate in body tissues with age. Senescent cells demonstrate characteristic morphological and metabolic changes. Cellular senescence is accompanied by large-scale spatial rearrangements of the nucleus leading to the formation of senescence-associated heterochromatin foci (SAHF) and senescence-associated distension of centromeres (SADS). A decrease in the level of the nuclear envelope component of lamin B1 leads to disruption of the chromatin structure and it is supposed to be involved in the mechanism of SAHF and SADS formation. Lamins take part in numerous regulatory cellular processes that influence cell proliferation, differentiation, and apoptosis. The aim of the work is to characterize the role of lamin B1 in replicative cellular senescence.

Methods and Algorithms: To generate primary human fibroblasts carrying the LMNB1 gene deletion we used CRISPR/Cas9 targeted mutagenesis. Guide RNAs were chosen using the CRISPR Design resource and cloned into PX459 vector. The efficiency of guide RNAs was determined using pEGxxFP system with following analysis by flow cytometry. Developed CRISPR/Cas9 targeted mutagenesis system was applied on LF1 primary lung fibroblasts cell strain.

Results: Genetic structures coding different guide RNAs to receive LMNB1 gene deletion by CRISPR/Cas9 were obtained. The efficiency of guide RNAs was determined by flow cytometry and the most effective were chosen for further work. Optimization of targeted mutagenesis conditions by the CRISPR/Cas9 method for removal of the coding region of the LMNB1 gene in primary human fibroblasts was carried out. A heterogeneous population of cells of primary human fibroblasts carrying the LMNB1 gene deletion was obtained. 